INPLASY

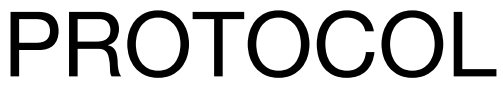

To cite: Almulihi et al.

Utilization, Efficacy, and Safety of Ketamine infusion as a

Single Sedative agent for adult and pediatric intubated

patients. Inplasy protocol

202210045. doi:

10.37766/inplasy2022.1.0045

Received: 09 January 2022

Published: 09 January 2022

Corresponding author:

QASEM ALMULIHI

qasem.almulihi@hotmail.com

Author Affiliation:

King Fahad University Hospital

- Saudi Arabia.

Support: No.

Review Stage at time of this submission: Completed but not published.

Conflicts of interest:

None declared.

\section{Utilization, Efficacy, and Safety of Ketamine infusion as a Single Sedative agent for adult and pediatric intubated patients}

\author{
Almulihi, Q1; Maghraby, N2.
}

Review question / Objective: Ketamine has both sedative \& analgesic properties and despite a lack of high-quality data, it is increasingly used for mechanically ventilated patients. However, combing more than 1 drug for sedation \& analgesia has a few issues, such as increasing the side effects, increased chance of medication error, more work on bedside nurses (preparation \& monitoring) in addition to possible cost. We aim to discuss and conclude the utilization, efficacy, and safety of ketamine infusion as a single sedative agent for Intubation of critically ill patients with or without comparison to another sedative agent (etomidate, propofol, thiopental, or midazolam).

Information sources: We used an extensive search strategy to cover several databases and clinical studies registries to identify all relevant literature. The initial search of the three electronic databases yielded and trial registries is 1322 potential studies. After exclusions based on titles and abstracts, the full texts of 60 studies were read for possible inclusion. A total of 7 studies remained for qualitative analysis. Figure 1 summarizes the results of our literature search.

INPLASY registration number: This protocol was registered with the International Platform of Registered Systematic Review and Meta-Analysis Protocols (INPLASY) on 09 January 2022 and was last updated on 09 January 2022 (registration number INPLASY202210045).

\section{INTRODUCTION}

Review question / Objective: Ketamine has both sedative \& analgesic properties and despite a lack of high-quality data, it is increasingly used for mechanically ventilated patients. However, combing more than 1 drug for sedation \& analgesia has a few issues, such as increasing the side effects, increased chance of medication error, more work on bedside nurses (preparation \& monitoring) in 
addition to possible cost. We aim to discuss and conclude the utilization, efficacy, and safety of ketamine infusion as a single sedative agent for Intubation of critically ill patients with or without comparison to another sedative agent (etomidate, propofol, thiopental, or midazolam).

Condition being studied: Adult and Pediatric intubated patients.

\section{METHODS}

Search strategy: An electronic search from PubMed/Medline and Google Scholar was conducted from their inception to 9th June 2021 without English language-based literature, Furthermore, we manually screened the cited articles from previous meta-analyses, systematic review, cohort studies including retrospective or prospective studies, and other review articles to identify any suitable studies.

Participant or population: Adult and Pediatric intubated patients.

Intervention: Ketamine infusion as a single sedative agent.

Comparator: Other sedative agents (etomidate, propofol, thiopental, or midazolam).

Study designs to be included: Cohort studies which is a type of longitudinal study.

Eligibility criteria: PICO: 1) P (Population): Adult or pediatric critically ill patient intubated in the pre- hospital setting or in the emergency department (ED) within 1 hour of arrival and connected to mechanical ventilator 2) I (Intervention): ketamine infusion as single agent; 3) C (Comparison): without comparison or with propofol, etomidate, thiopental, midazolam, Methohexital, Dexmedetomidine, Fentanyl; 4) 0 (Outcome): utilization, efficacy, and side effects representing safety for use.

Information sources: We used an extensive search strategy to cover several databases and clinical studies registries to identify all relevant literature. The initial search of the three electronic databases yielded and trial registries is 1322 potential studies. After exclusions based on titles and abstracts, the full texts of 60 studies were read for possible inclusion. A total of 7 studies remained for qualitative analysis. Figure 1 summarizes the results of our literature search.

Main outcome(s): Utilization, Efficacy, and Safety of Ketamine infusion as a Single Sedative agent for adult and pediatric intubated patients.

Quality assessment / Risk of bias analysis: Two reviewers independently searched electronic databases. Studies searched were exported to the EndNote Reference Library software version 20.0.1 (Clarivate Analytics) and duplicates were screened and removed. Data extraction and quality assessment of included studies was done simultaneously and independently by three reviewers. Newcastle-Ottawa Scale (NOS) was used to assess the quality of the cohort studies. NOS score 1-5 were considered as high risk for bias, 6-7 was moderate and score $>7$ was considered low risk of bias.

Strategy of data synthesis: The results were formed by using a qualitative analysis. We intended to summarize the findings of the articles to synthesis the results. It was selected as it incorporated the results from all articles and highlights all the differences and similarities between the study findings. Since the included studies showed heterogeneity among their evaluation criteria and study results, performing a meta-analysis was not considered suitable, as it would have yielded potentially nonsignificant and misleading results.

Subgroup analysis: There was no Subgroup analysis.

Sensitivity analysis: Since the included studies showed heterogeneity among their evaluation criteria and study results, performing a meta-analysis was not considered suitable, as it would have 
yielded potentially non-significant and misleading result.

Language: English.

Country(ies) involved: Saudi Arabia.

Keywords: Ketamine, mechanical ventilations, continuous sedation, critically ill patient, single agent.

Contributions of each author:

Author 1 - Qasem Almulihi.

Email: qasem.almulihi@hotmail.com

Author 2 - Nisreen Maghraby.

Email: nhmaghraby@iau.edu.sa 